\title{
Electron Beam for LHC
}

\author{
Mieczyslaw Witold Krasny, \\ LPNHE, University Pierre et Marie Curie, Paris, France
}

Abstract: A method of delivering a monochromatic electron beam to the $\mathrm{LHC}$ interaction points is proposed. In this method, heavy ions are used as carriers of projectile electrons. Acceleration, storage and collision-stability aspects of such a hybrid beam is discussed and a new beam-cooling method is presented. This discussion is followed by a proposal of the Parasitic Ion-Electron collider at LHC (PIE@LHC). The PIE@LHC provides an opportunity, for the present LHC detectors, to enlarge the scope of their research program by including the program of electron-proton and electron-nucleus collisions with minor machine and detector investments.

\section{Introduction}

The LHC detectors will explore the high energy frontier of proton-proton, protonnucleus and nucleus-nucleus collisions. From the perspective of the Standard Model the rôle of proton and nuclear beams is confined to that of carriers of virtual but Lorenz-frozen, Wide-Band-Beams (WBBs) of the basic, point-like building blocks of matter, and of their interaction quanta.

Hadronic colliders are optimal for generic exploration of interactions of a large variety of the Standard Model particles, over a large momentum range, which extends up to the momentum of the carriers of partonic beams. Their merits are complementary to those of electron-positron colliders, which employ better-controlled, but less-luminous Narrow-Band-Beams (NBBs) of the Standard Model particles, colliding in a cleaner environment.

The electron-proton (electron-nucleus) colliders can neither compete with the hadronic colliders in exploring the small-distance frontier of interactions of the basic constituents of matter, nor with the electron-positron colliders in the measurement precision. Nevertheless, they are very useful for a precise mapping of the emittance and momentum distributions of partonic WBBs generated by colliding hadrons. 
Such a mapping will remain indispensable as long as the quantitative predictive power of the Standard Model will be confined to the perturbative calculation methods. It must involve unfolding of the effects of space-time confinement of virtual bunches of partonic beams, and the effects due to strongly-interacting medium in which they are colliding.

The experimental programme of the LHC collider could be significantly enriched, and the measurement precision could be increased, by adding a monochromatic electron beam to the LHC beam inventory, in particular, if such a beam could be delivered to the Interaction Points (IPs) of the LHC detectors, for collision with the LHC hadronic beams. If provided, it could allow the LHC measurements to be largely independent of the partonic-distribution-function (PDF) extrapolation-technology. This technology assumes the validity of the factorization (WBB-invariance) of the Standard Model hard processes over the full kinematic domain, including regions where it has never been tested. In addition, it relies upon the phenomenological modeling of the energy and of the nuclear dependence of partonic distribution and upon the energy-independent partonic-beam emittance.

If the electron-proton and the electron-ion collisions could be observed and recorded by the LHC detectors concurrently with hadronic ones, they would provide, for the latter, a high-precision control of the time dependent detector calibration. Their studies could allow for a precise, detector-specific unfolding of the non-perturbative QCD effects, which are present in the relation between the fastmoving coloured constituents of matter and the observed jets. A monochromatic electron beam accompanying partonic WBB in hadronic colliders could thus play a similar rôle to that of a monochromatic electron beam which produces the partonic NBB in the electron-positron colliders.

The merits of the electron beam must be weighted against an extra cost and against a possible interference of the corresponding electron-proton (electronnucleus) collision programme with the standard LHC programme. In this paper, I shall assume that the electron beam will not be accepted unless it is delivered with no extra cost. In addition, I shall require the bunch structure of the electron beam to be identical to that of the standard LHC beam such that the LHC detectors could register the electron-proton (electron-ion) interactions without any hardware modification, in particular with no readjustments of the detector electronics. Moreover, storage of the electron beam must not degrade the quality of the standard LHC beams, and its presence must not be in conflict with the foreseen operation mode of the LHC collider. The electron beam must be, thus, fully parasitic. Last, but not least, I shall require the energy of the electron beam to be optimal for a high precision mapping of partonic WBBs at the LHC, in the most difficult, small $x_{B j}$ region, using the unmodified $\mathrm{LHC}$ detectors.

The goal of this paper is to propose a method of delivering a monochromatic electron beam to the IPs of the LHC experiments satisfying all the above require- 
ments. The present paper summarizes the preliminary feasibility studies of the proposed method. The technical details will be presented elsewhere.

\section{The idea}

The basic idea is very simple. It consists of using heavy ions as the carriers of the monochromatic beam of electrons. Electrons are attached to the atomic nucleus by means of Coulomb attraction and they form a partially ionized atom. The distance between the electrons and the atomic nucleus, even in the case of lowest energy states of the highest-Z atoms, is significantly larger than the range of strong interactions. This allows the electrons and the nucleus to be considered as free particles in large momentum transfer collisions. The electron collisions, at the energies of LHC collider, are thus almost unperturbed by the presence of the nucleus. ${ }^{1}$

An atomic nucleus carrying $k$ electrons can be stored at the energy which is limited by the maximum magnetic field of the bending magnets:

$$
E_{\text {ion }}=E_{Q} \times(Z-k) .
$$

The $E_{Q}$ represents the energy-per-charge machine parameter, related to the strength of the magnetic field, and $Z$ represents the number of protons in the nucleus. Each electron carries the longitudinal momentum of:

$$
p_{e}=M_{e} / M_{\text {ion }} \times E_{Q} \times(Z-k),
$$

where $M_{e}$ and $M_{i o n}$ are, respectively, the electron and the ion masses. Note, that smearing of both the longitudinal and the transverse momentum of the electron, due to its orbital motion, contrary to the Fermi motion of the nucleons within a nucleus, can be neglected for the LHC collider energies. The electron beam can be considered, thus, with a high precision as monochromatic.

If, for example, two electrons would be transported to the LHC IPs by the lead ions to collide with the LHC protons at the top energy, then the maximum CM-energy of the electron-proton collisions would be $\sqrt{s} \approx 200 \mathrm{GeV}$. This value is close to the energy of the HERA collider. If such a collision-configuration would be realized at the RHIC collider at the BNL, the CM-energy of the electron-proton collisions would be smaller than those of the SLAC fixed target deep-inelastic experiments, where the partonic picture of nucleons was established.

As far as the achievable electron-proton collision energy is considered, the LHC is, thus, the first hadronic collider, in which interactions of electrons carried by the ions could be efficiently used to map, over a large kinematic domain, partonic

\footnotetext{
${ }^{1}$ The electromagnetic radiative corrections due to the soft photon emission have to be calculated assuming an effective cut-off reflecting the quantum structure of atomic levels.
} 
structure of nucleons and nuclei. A large asymmetry of the energies of the electron beam, $E_{e} \approx 1.5 \mathrm{GeV}$, with respect to the proton beam, $E_{p}=7000 \mathrm{GeV}$, makes such a collision-scheme particularly well suited for mapping of small $x_{B j}$ region using the existing LHC detectors. As an example, for collisions of massless partons, carrying a fraction of $2 \times 10^{-4}$ of the nucleon momentum, with such an electron beam, the electron-parton collision CM-frame is at rest with respect to the laboratory frame of the LHC detectors. This configuration allows for an optimal measurement of deep inelastic scattering kinematics in the precise barrel sections of the existing detectors.

There are two ways of delivering partially ionized atoms to the LHC ring. The first one is to use an electron capture foil in the transfer line between the SPS accelerator and the LHC storage rings. This method is not efficient and will not be discussed in this paper. The second, advocated method consists of modifying the standard stripping sequence of the LHC ion beams. For example, stripping from the the $\mathrm{Pb}^{54+}$ charge state to the $\mathrm{Pb}^{82+}$ charge state (fully-stripped ion), foreseen at the transfer line between the PS and the SPS, would have to be replaced by a stripping procedure which leaves $k$ electrons. Such a beam would have to be transfered to the SPS and, subsequently, to the LHC and accelerated to the top energy. The optimal value of $k$, discussed in more details in the following sections, should represent an optimal trade-off between the acceleration and the collision stability of the beam at the largest possible energy - requiring $k$ to be be small, and the largest possible equivalent current of the electron beam - requiring $k$ to be large. In addition, the stripping loses due to the acceptance of the only one charge state at each stripping stage, have to be minimized by limiting the number of stripping stages and by stripping on a shell-by-shell basis.

The idea of using ions as carriers of a monochromatic beam of electrons seems to be rather straightforward. What is far from being straightforward is how to form, accelerate, store and collide such hybrid beams at the top LHC energy.

The beams of partially-stripped ions are particularly fragile and have to be treated with a special care. Exposed to the strong magnetic field of the LHC bending magnets, they have to survive a static electric field, which is 20 times stronger than that binding an electron on the K-shell of the hydrogen atom. When accelerated to the top LHC energy, the temperature of bunches of partially-stripped ions must be stabilized, such that the ions are not exposed to temperatures exceeding their characteristic ionization temperatures. To be stored over a long time at the top energy, they need to be strongly bound to minimize losses due to interactions with the residual gas in the machine. The luminosity of colliding beams of partially-stripped ions should be sufficiently high to allow for a satisfactory statistical precision of mapping of the WBBs at the LHC, but not too high for an excessive beam-collision stripping rate. The last two constraints must be give a special attention in the case of superconducting storage rings. Even if one electron is lost, the magnetic rigidity of the carrier ion is changed and the ion hits the beam pipe within a fraction of a 
microsecond. If the rate of the electron losses is high, then such desynchronizing of their parent ions may cause quenching of the magnets.

At first sight, dealing with such beams in superconducting storage rings seems to be hopeless. This is presumably the reason why it has never been considered. All the above constraints impose, indeed, very stringent requirements on what beams can be formed, and how they must be accelerated, stored, and collided. These requirements are discussed in the following sections.

\section{Survival of partially-stripped ions in the LHC lattice}

Beam particles circulating in the LHC storage rings are exposed to a strong magnetic field of superconducting dipoles. The bending field $B$, when viewed in the rest frame system of stored bunches, is equivalent to an electric field $E$ with the strength of:

$$
E=\gamma \times \beta \times c \times B
$$

and of the direction perpendicular to that of the magnetic field. The dipole field of 8.4 Tesla is equivalent, for ultra-relativistic beam particles moving with velocity corresponding to the Lorenz- $\gamma$ of 3000, to the electric field of a strength: $E=7.3 \times$ $10^{10} \mathrm{~V} / \mathrm{cm}$. The field of such strength will not only modify the atomic energy levels of partially-stripped ions (Stark effect) but, may, in addition, strip the electrons.

Rausch and Traubenberg [1], while investigating the effect of the electric field on the atomic Balmer lines, observed that each spectral line ceased to exist above a certain field strength. The suppression of spectral lines was the first observation of ionization of atoms by strong electric fields.

The quantum-mechanical description of the mechanism of quenching of spectral lines has been presented in the work of Lanczos [2] and Oppenheimer [3]. Spectral lines are suppressed if the probability of ionization of the initial excited state of an atom is greater than the probability for its radiative de-excitation. Since the atomic de-excitation time is typically of the order of $10^{-8}$ seconds, the quantum-mechanical tunneling probability of an atom to lose over an atomic unit time $\left(2.4 \times 10^{-17}\right.$ seconds $)$ one electron must be smaller than $10^{-9}$. This limit determines, for each atomic energy level, the maximum field strength, at which an atom in a given charge state can survive or, conversely, for a given field strength, which charge states of partially-stripped ions can be stored at a given energy.

The results of the calculations, which are relevant for the present paper, are summarized below. Light ions, up to oxygen, can be stored in the LHC ring only in the fully-stripped charge state. High- $Z$ partially ionized atoms can survive the LHC magnetic field in a wide range of charge states, provided that the binding energy of the least-bound electron is kept above $E_{b} \approx 0.9 \mathrm{keV}$. 
In the following, I shall restrict the discussion to the case of lead ions as potential candidates of carriers of the electron beam. The main reason is that the fullystripped lead-ion beam is already foreseen in the LHC experimental programme. ${ }^{2}$ I shall also restrict the discussion to the charge state $\mathrm{Pb}^{80+}$ which maximizes both the electron beam energy and intensity. In such a charge state, two electrons remain attached to the $P b$ nucleus. These electrons can be safely transported not only on their K-shell orbit, but also in a wide range of excited states, provided that the energies of their excitations will not exceed the critical one. This critical energy for $P b^{80+}$ turns out to be equivalent to the binding energy of the excited $n_{\max }=10$ state of the $Z=82$, single-electron Rydberg atom.

The $\mathrm{Pb}^{80+}$ ions can be thus considered as robust candidates for the carriers of electrons resistant to various environmental perturbations, which may result in their excitations.

\section{Survival of bunches of partially-stripped ions}

\subsection{Temperature}

Ions are accelerated and stored in bunches. The evolution of bunch sizes is driven by the RF-system and by the beam focusing optics. The motion of the beam particles within their bunches can be characterized by the bunch temperature. ${ }^{3}$ In the following, I shall concentrate on the transverse motion of the beam particles. The longitudinal motion and its coupling to the transverse motion is omitted in this paper for simplicity.

The transverse temperature of bunches can be expressed in terms of: the normalized emittance of the beam, $\epsilon_{N}$, the average (over the ring) transverse size of the bunches, $\sigma_{h, v}$, the mass of the ions, $M_{i o n}$, and the ion velocity $\beta$ as:

$$
k T_{h, v}=M_{\text {ion }} \times c^{2} \times \beta^{2} \times\left(\epsilon_{N} / \sigma_{h, v}\right) .
$$

The critical issue of accelerating bunches of partially-stripped ions becomes apparent when the temperature of the LHC bunches is calculated. The transverse temperature at the injection to the LHC reaches equivalent energy of $1 \mathrm{MeV}$ and rises linearly with increasing $\gamma$ of the beam. Even if initially carried on the K-shell of the highest-Z ions, the electrons are stripped at such temperatures.

\footnotetext{
${ }^{2}$ All the arguments and conclusions presented in this paper hold if lead ions are replaced by gold ions (the BNL heavy-ion beam).

${ }^{3}$ Expressing the beam particle motion in terms of an equivalent temperature requires the average collision time between the beam particles to be small with respect to the acceleration and the storage times. Note that, in this context, the frequency of the intra-beam collisions of partiallystripped ions is significantly higher than that of the protons.
} 
A method of stabilizing temperatures of bunches below the ionization temperature must be invented for such a beam. The method, which is proposed here is based upon a concept of the controlled, iso-thermal heat evaporation by light emission. This method is illustrated in Fig. 1 and explained in the following.

\subsection{Temperature evolution of bunches of fully and partially- stripped ions}

The energy levels of an atom with the nucleus charge $Z$ can be subdivided into two subsets: the energies below and above $E\left(n_{\max }\right)$. The $E\left(n_{\max }\right)$ is the energy of the atomic level above which the ion is spontaneously ionized by the machine B-field. If an electron carried by the lead ion on its K-shell ( $n=1$ state) is excited by an inelastic collision to the $n>n_{\max }=10$ state, it will be promptly stripped off.

\section{Acceleration of bunches of partially stripped ions}

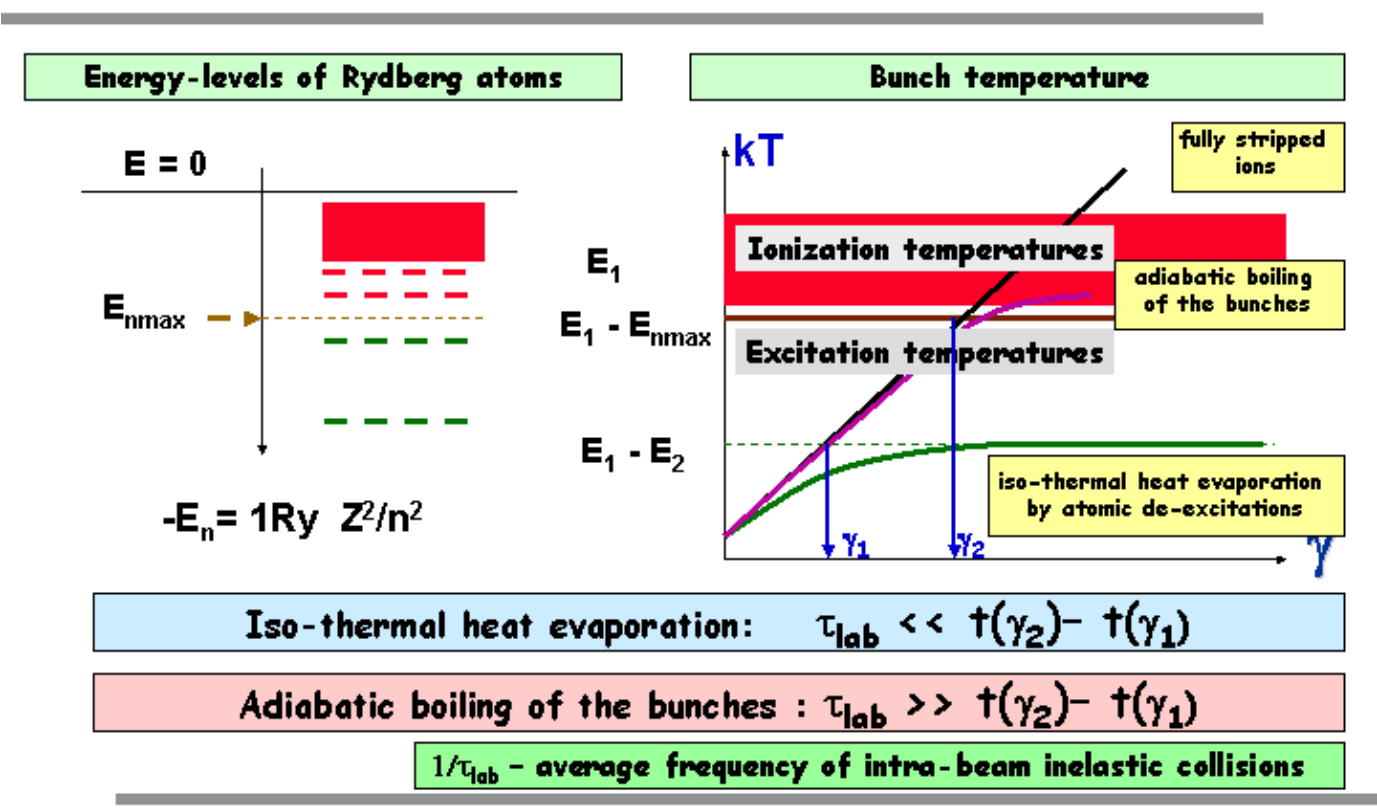

Figure 1: The temperature evolution of the accelerated bunches of partially-stripped ions.

At high beam energies, the temperature of the bunches of fully-stripped ions 
increases with the Lorenz $\gamma$ factor of the beam. This dependence is analogous to an increase of the temperature of an ideal gas, in the adiabatic change of the gas volume.

\section{Stabilization of bunch temperatures}

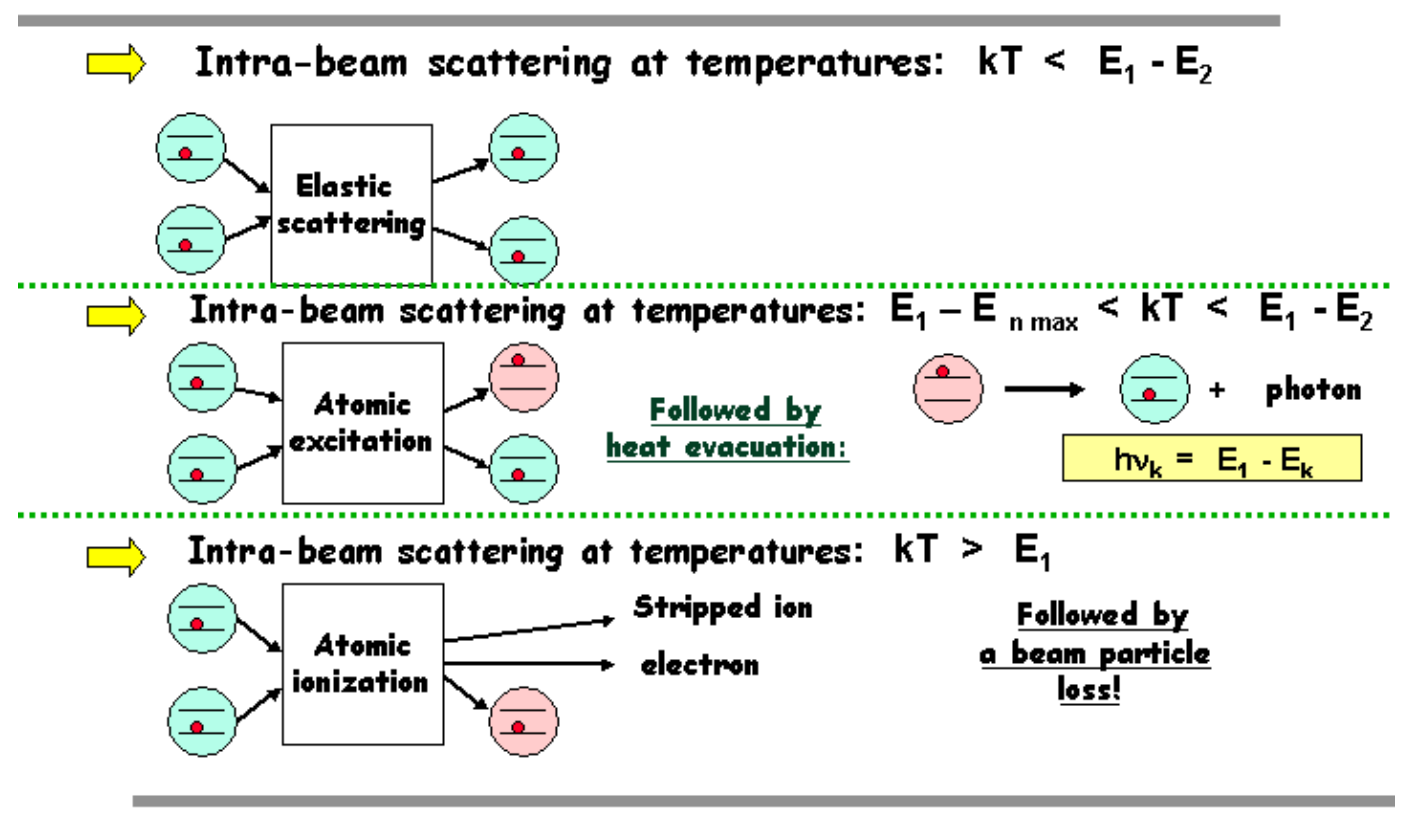

Figure 2: Intra-beam collisions of partially-stripped ions.

The temperature evolution of the bunches of partially-stripped ions will be affected by their atomic energy-level structure in a way which is illustrated in Fig. 2. At the early acceleration stage, when the bunch temperature is lower than the difference between the ground state and the first excited state of the ion, the beam collisions are solely elastic. In this regime, the temperature evolution of the beam of partially-stripped ions will be the same as that for the fully-stripped ion beam. At the acceleration stage, when the bunch temperature becomes larger than the difference between the ground, and the first excited state of an ion, but smaller than the difference between the ground state and the $n_{\max }$ state, ions will collide both elastically and inelastically. The inelastic collisions will lead to atomic excitations followed by photon emissions. Finally, if the temperature of bunches will be allowed to exceed the critical ionization level (the difference between the ground state and 
the $n_{\max }$ state) the inelastic collision of partially ionized atoms may lead to their full ionization.

\subsection{Fast acceleration of bunches of partially-stripped ions}

Given the above picture, two methods of accelerating bunches of partially-stripped ions are proposed. The first one consists of fast acceleration of the beam. The acceleration gradient $d \gamma / d t$, in this method, is required to be sufficiently large such that, at any given moment of the beam acceleration, within the time interval between reaching the critical ionization temperature and reaching the top beam energy, the bunches are not allowed to thermalize. Note, that the termalization time increases linearly with the Lorenz $\gamma$ factor. This method employs an effective "dilution" of the gas of overheated ions due to slowing down the intra-beam collisions as observed in the accelerator rest frame. The resulting bunches will be, however, unstable - the inelastic intra-beam collisions at the top energy will lead to stripping of electrons and the beam will eventually be lost unless it is quickly cooled down at the LHC top energy. The Doppler cooling [4] may be used for this purpose. Its efficiency would be significantly enhanced if the atomic transitions rather than the nuclear excitation transitions are used. It is interesting to note, that the LHC is the first collider where such a cooling can be done at the top beam energy using conventional, high power UV lasers. Note, that the $\gamma$ factor at the top LHC energy, driving the Doppler gain of the energy of the laser light, becomes sufficiently high to compensate the $Z^{2}$ increase of the binding energy of atomic levels of partially-stripped ions.

The fast acceleration method complemented by the laser cooling at the top LHC energy is indeed very attractive but requires dedicated investments. Such investments are unlikely be made at CERN solely for the purpose of cooling of partially-stripped ion beams. They may, however, be driven by the side gains of such a cooling scheme (e.g., creation at CERN of a luminous, monochromatic and highly collimated X-ray beams in the MeV energy range).

In order to avoid adiabatic boiling of the bunches at the collision energies, without external beam cooling, an alternative acceleration method is proposed below. In this method, the excessive heat of adiabatically compressed bunches is evacuated iso-thermally by means of radiative evaporation. The acceleration rate of partially-stripped ions must be maximized at low energies to avoid the effects driving the effective increase of the bunch sizes. The ions must then be stripped as early as possible to the final beam-collision charge state. The crucial point of the method is that, at the critical acceleration phase of the beam in its final beamcollision charge state, when the bunch temperature reaches the atomic excitation temperature $\left(\gamma_{\text {crit }}=\gamma\left(T=\left(E_{1}-E_{2}\right) / k\right)\right)$, the process of acceleration must be slowed down to allow for gradual heat evaporation. The acceleration rate $d \gamma / d t$ should be reduced to such a value that at any moment of the beam acceleration 
process, spanning the time interval between reaching the critical ionization temperature and reaching the top beam energy, the ions are kept at thermal equilibrium. Under this condition, inelastic collisions of ions could effectively convert the excessive kinetic energy of the most energetic ions (occupying the tail of the Maxwell distribution) into the energy of their excitation. The energy absorbed by the atomic excitations will be subsequently evaporated by the ion-de-excitation photons. In the model of asymptotically-slow acceleration, with no other dissipative processes present, the asymptotic beam temperature should stabilize at the temperature of $T_{\max }=\left(E_{1}-E_{2}\right) / k$.

\subsection{Numerical exercise}

The condition for the iso-thermal heat evaporation can be quantified by using an acceleration model specified in terms of the following parameters:

- number of $P b^{80+}$ ions per bunch - $N_{\text {ions } / \text { bunch }}=10^{7}$,

- normalized beam emittance - $\epsilon_{N}=1.5 \times 10^{-6} \mathrm{~m}$,

- average $\beta$ over ring circumference $-\langle\beta\rangle=67 \mathrm{~m}$,

- bunch length in the storage-ring rest frame $-L_{b u n c h}=7.5 \mathrm{~cm}$,

- the atomic excitation cross section - $\sigma_{\text {excit }}=10^{-18} \mathrm{~cm}^{2}$.

In such a model, the temperature of lead bunches is expected to stabilize at the level of $68 \mathrm{keV}$, provided that following the fast acceleration phase, up to $\gamma \approx 10$, the acceleration rate is reduced to the value of $d \gamma / d t<0.8 /$ minute. In this model, slowing down of the acceleration rate must take place at the SPS. If the above model is applied to the acceleration of bunches of the gold ions at BNL, the onset of the temperature stabilization should be observed in AGS, where bunches of $A u^{77+}$ carrying two unstripped electrons are accelerated. The processes of temperature stabilization is expected to reduce the normalized emittance of the beam. Thus, the

emittance of the top-energy $A u^{77+}$ beam in AGS should be smaller than that of the beam fully-stripped $A u^{79+}$ ions.

\subsection{Merits of the temperature stabilization}

If the temperature stabilization is confirmed experimentally, the low temperature bunches of partially-stripped ions could be accelerated up to the top LHC energy with residual ionization losses due to stripping of the ions by the intra-beam scattering. Moreover, the proposed cooling technique could be employed to improve the beam quality for the standard LHC collision programme which uses fully-stripped 
ions. In order to profit from the temperature stabilization, the stripping of the two last electrons of the lead ions would have to be postponed up to the beam transfer between the SPS and the LHC. The effective reduction of the beam emittance could allow for the reduction of the beam current at fixed luminosity. Equivalently it could allow for increasing the $\beta^{*}$ value at fixed beam current and luminosity. But, first of all, it could allow to reduce the beam envelope providing cleaner beams.

\section{The lifetime of the beam of partially-stripped ions in the LHC collider}

\subsection{Ionization processes}

If thermally-stable, partially-stripped ion bunches can be stored at the LHC, their lifetime will be governed by the rate of collisions of ions with the residual gas in the ring. The beam losses will be dominated by the ionization processes in which an electron is stripped-off from the carrier ion by a residual gas molecule. In the collision phase, the electrons carried by the ions will also be stripped in the beambeam collisions. While the beam-gas losses can be reduced by improving the machine vacuum, the beam-beam collision losses represent an irreducible limit governed by the machine luminosity.

The K-shell ionization of ultra-relativistic ions has been discussed extensively by R. Anholt and U.Becker [5]. The ionization cross section can be expressed as the sum of the three contributions: the Coulomb , the transverse, and the spin-flip ionization. The Coulomb cross section does not depend upon the collision energy. The contribution of transverse ionization is most important in collisions with large impact parameters. It increases logarithmically with increasing $\gamma^{2}$. The spin flip contribution can be safely neglected in the following discussion.

The theory of Anholt and Becker has been successfully tested at $\gamma=168$ by measuring $\mathrm{Pb}^{81+}$ ionization cross section in gaseous $\mathrm{Ar}, \mathrm{Kr}$ and Xe targets [6]. The predicted cross sections were found to be slightly larger that the measured values. Thus, there is a safety margin in applying this theory to calculate the lifetime of the beam of partially-stripped ions at the LHC, by extrapolating the existing measurements to different targets and higher collision energy. Such extrapolations must take into account the difference between the ionization of the projectile particles by atoms (verified experimentally), and by fully or partially-stripped ions. The ion charge screening corrections, which flatten the energy dependence of the ionization cross-section, become small in the limit of $Z_{c}>>Z_{t}$, where $Z_{c}$ is the charge of the carrier of the electrons and $Z_{t}$ is the charge of the target. In this limit K-shell-electron wave functions are "localized" within a small transverse surface, with respect to the distances between the target electrons and target nucleus and 
projectile electrons "see" almost-unscreened target nucleus.

Another important aspect of the R. Anholt and U.Becker theory, which will be employed in the subsequent sections, is the dependence of the ionization cross section on $Z_{c}$ and $Z_{t}$. Neglecting logarithmic contributions, both the Coulomb and the transverse cross sections are proportional to $\left(Z_{t} / Z_{c}\right)^{2}$.

\subsection{Beam-gas collisions}

The beam-gas lifetime can be expressed in terms of the ionization cross section $\sigma_{i}$, and the density $\rho_{i}$ of the molecules of type $i$ remaining in the beam pipe:

$$
\tau^{-1}=\Sigma_{i}\left(\sigma_{i} \times \rho_{i} \times c\right)
$$

The calculations presented below were made for the K-shell ionization. This is fully justified as, even for the bunch temperatures, where the Boltzmann proportion of atoms in various excited states has to be taken into account, the lifetime of these excited states is sufficiently short for this approximation to be valid - independently of the temperature stabilization efficiency.

The calculated ionization cross sections for the residual gas molecules listed e.g in [8] are large. They span the range of $30-2000$ barns depending on the type of the molecules (the lowest cross section corresponds to the $H_{2}$ and the highest to the $\mathrm{CO}_{2}$ molecules). The beam-loss cross sections for molecules containing high $Z$ atoms are significantly higher than those for fully-stripped ions. Therefore, the vacuum requirements for partially stripped ions are the most demanding ones. The maximum allowed densities of gas molecules to keep alive the beam of $\mathrm{Pb}^{80+}$ ions over $\tau=10$ hours were found to be 7 to 200 times lower than the corresponding maximum allowed concentration for storing fully-stripped lead ions [7]. The span of these factors reflects the underlying feature of charge coherent processes, which make partially-stripped ion beams singular - the strong $Z_{t}^{2}$ dependence of the ionization cross sections. Therefore, in order to achieve long stores of partially-stripped ions, it is indispensable to reduce, as much as possible, the contribution of gasses containing high $\mathrm{Z}$ atoms, in particular the density of the $\mathrm{CO}$ and the $\mathrm{CO}_{2}$ molecules.

The question if the long stores of $\mathrm{Pb}^{80+}$ ions are feasible at the LHC will remain open until a sufficient experience of the LHC operation is acquired. If the estimation of the concentration of molecules in the IPs of the LHC experiments by Rossi and Hilleret [8] (scenario after machine conditioning) will be confirmed by the real LHC operation conditions, the ten-hour-long stores of partially-stripped lead ions will be feasible. The concentration of the $\mathrm{CO}_{2}$ molecules is critical. Their predicted density [8] is only two times smaller than the maximum allowed density for the ten-hour-long store of the $P b^{80+}$ beam, leaving only a marginal safety factor. 


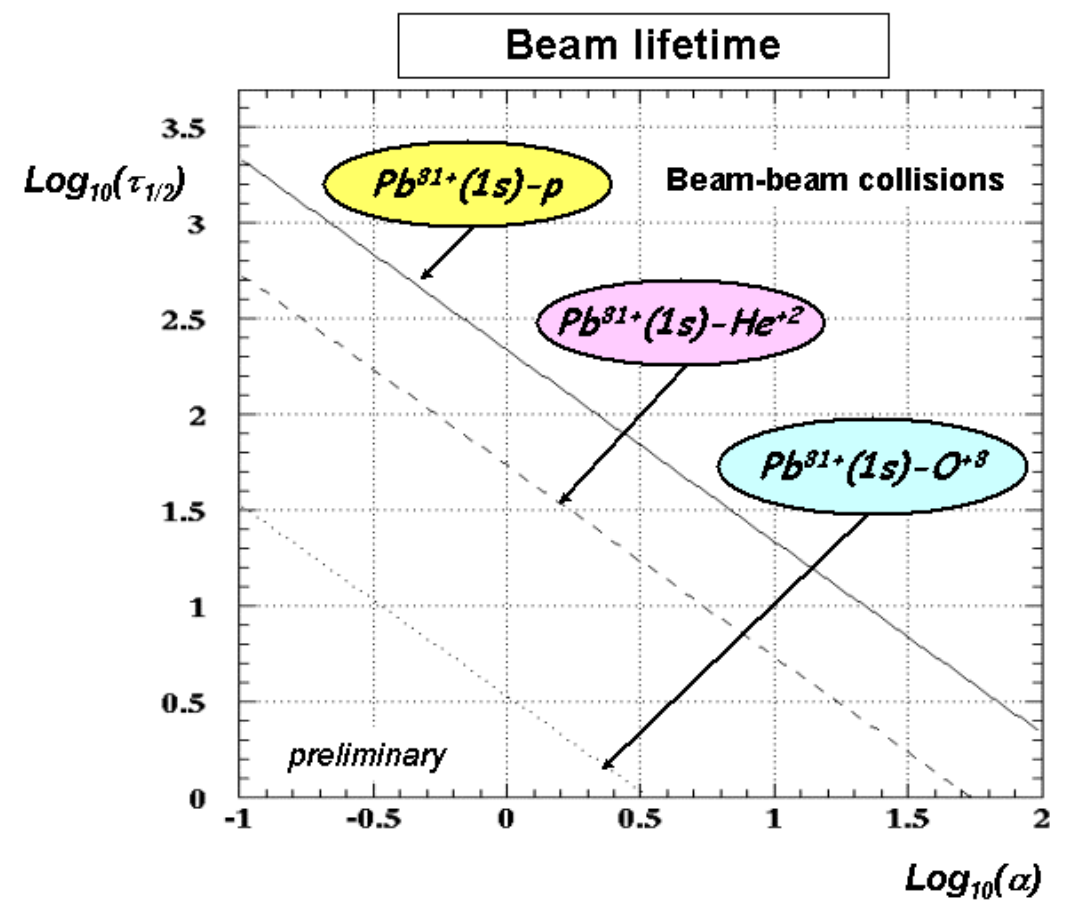

Figure 3: The collision lifetime of the $P b^{81+}$ ions as a function of the electronproton collison luminosity. For the $\mathrm{Pb}^{80+}$ ions the luminosity increases and the lifetime decreases by a factor of 2 .

Note, however, that if the vacuum in the arcs, as it is expected, will be better than that in the interaction regions, for which the predictions [8] have been made, the beam lifetime will increase.

\subsection{Beam-Beam collisions of partially-stripped ions}

From the above discussion, it is evident that only the high- $Z$ carriers of the electrons can be stored at LHC for sufficiently long time. The question, which is addressed in this section is: what is the luminosity lifetime if the beams of partially-stripped ions are colliding with each other?

At first symmetric collisions are considered (i.e., those of the same ions circulating in the LHC rings in the opposite directions). The evolution of the number of ions can be calculated using the following equation:

$$
-1 / N \times d N / d t=n_{I R} \times L \times \sigma_{i o n} /(k \times N)=\lambda,
$$


where $N$ is the number of ions per bunch, $k$ is the number of bunches, $\sigma_{i o n}$ is the ionization cross section and $L$ is the luminosity.

The luminosity evolves with time according to:

$$
L(t)=L(0) /(1+\lambda t)^{2} .
$$

Using the Anholt and Becker theory for $\sigma_{i o n}$ and assuming the standard machine parameters: $N=9 \times 10^{7}, L=10^{27} \mathrm{~cm}^{-2} s^{-1}, k=608$, and $n_{I R}=2$, the calculated luminosity lifetime is found to be smaller than one minute. Thus, colliding of beams with the same $\mathrm{Z}$ is excluded.

\subsection{Allowed collision schemes}

The results presented in the previous section demonstrate clearly, that even if an effective beam of electrons carried by high-Z ions can be stored in the LHC, such a beam cannot be used for the high-luminosity collisions of electrons with high$\mathrm{Z}$ nuclei. Indeed, the ionization cross section driving the beam losses is huge. It reaches a fraction of $\mathrm{Mb}$ for the highest- $Z$ ions at the maximum LHC energy. This result does not, however, exclude other possible collision schemes.

Indeed, the drop of the ionization cross section for Z-asymmetric collisions, which is governed by the $\left(Z_{t} / Z_{c}\right)^{2}$ dependence is impressive. For example, the ionization cross section of $\mathrm{Pb}^{80+}$ ions colliding with the lead ion beam is almost $10^{4}$ higher than that for collisions with protons. The asymmetric collisions in which two beams of unequal magnetic rigidity are colliding are thus allowed - the only consequence is that the effective energies of ions circulating in the opposite beams will be unequal. The usefulness of the beam of partially-stripped ions is thus restricted to the highly asymmetric collisions in which the electrons, attached to the highest-Z ions, collide with protons or fully-stripped low-Z ions.

In Fig. 3, the logarithm of the luminosity lifetime is plotted against the logarithm of the luminosity for collisions of $P b^{81+}$ ions with protons and low- $Z$, fully stripped ions. The luminosity is expressed units of $10^{27} \mathrm{~cm}^{-2} \mathrm{~s}^{-1}$ (the $\mathrm{Pb}-\mathrm{Pb}$ collision units). For example, if one requires the lifetime to be ten hours, then the luminosity which can be achieved in the $\mathrm{Pb}^{81+}$ - proton collisions will reach the value of $0.4 \times 10^{29} \mathrm{~cm}^{-2} \mathrm{~s}^{-1}$. Collisions with light ions, with similar luminosity lifetime, will also be possible, albeit at the price of reduced luminosity. This reduction will be partially compensated by the A-times increase of the cross section for point-like processes (not taken into account in Fig. 3). The rate of point-like collisions of electrons with nuclei scales as $A / Z^{2}$. For example, the rate of the electron-oxygen point-like collisions will be reduced by a factor 4 with respect to the electron-proton collision-rate at the same beam life-time limit.

It is worthwhile to note, that the integrated electron-proton luminosity used

in the first measurements of the structure functions at small $x$ at HERA [9] can be 
be collected in two, ten-hour-long $P b^{80+}-p$ collision runs at LHC.

\section{The PIE@LHC collider}

\subsection{The running mode}

The physics programme of the electron-proton and the electron-nucleus collisions, must be introduced in a non-invasive way. The merits of this proposal must be demonstrated while avoiding any interference with the canonical running mode of the LHC collider and while avoiding any interference with optimal tuning of the LHC detectors for the standard LHC hadronic-beam programme.

Within the canonical LHC running scenario, it is very likely, that the first $\mathrm{Pb}$ $\mathrm{Pb}$ collision runs will be followed by the proton- $\mathrm{Pb}$ runs. If, at this stage, the machine vacuum is controlled to the level discussed in section 5.2 , the beam of fully-stripped lead ions could be replaced by the beam of partially-stripped lead ions. Collisions of electrons could be recorded in such runs together with proton $-\mathrm{Pb}($ ion $-\mathrm{Pb})$ collisions.

Those of the LHC experiments, which would not be interested in the PIE@LHC collider programme, could ignore the parasitic electron-proton collisions altogether. The only noticeable difference for them will be a residual reduction of the CM-energy of the proton $-P b($ ion $-P b)$ collisions by an $r=\sqrt{80 / 82}$ factor. Since the rate of proton - electron (ion - electron) collisions will be significantly lower than that of the proton $-\mathrm{Pb}$ collisions, their triggering, filtering and registering would consume only an insignificant fraction of the data collection resources, which may remain devoted to recording proton $-\mathrm{Pb}$ collision events.

The only required action of those of the LHC experiments which will be willing to analyze the electron-proton collision events is to include a dedicated trigger capable to select a "topologically spectacular" electron-proton collision. The trigger and the data-acquisition model discussed in [10] anticipates already a clash-less absorption of the PIE@LHC collider programme in a way which is transparent to the mainstream physics programs of the present LHC detectors.

\subsection{The collider parameters}

The parameters of the PIE@LHC collider for electron-proton collisions are summarized below:

- CM-energy - $200 \mathrm{GeV}$,

- luminosity - $0.4 \times 10^{29} \mathrm{~cm}^{-2} \mathrm{~s}^{-1}$,

- number of bunches - 608 , 
- number of ions per bunch $-9.4 \times 10^{7}$,

- number of protons per bunch $-4 \times 10^{9}$,

- transverse emittance - $\leq 1.5 \mu m$,

- $\beta^{*} \geq 0.5 m$ (depending on the reduction factor of the beam emittance).

\subsection{The kinematic domain}

The kinematic $\left(x, Q^{2}\right)$ domain which is accessible for the PIE collider is shown in Fig. 4 and in Fig. 5.

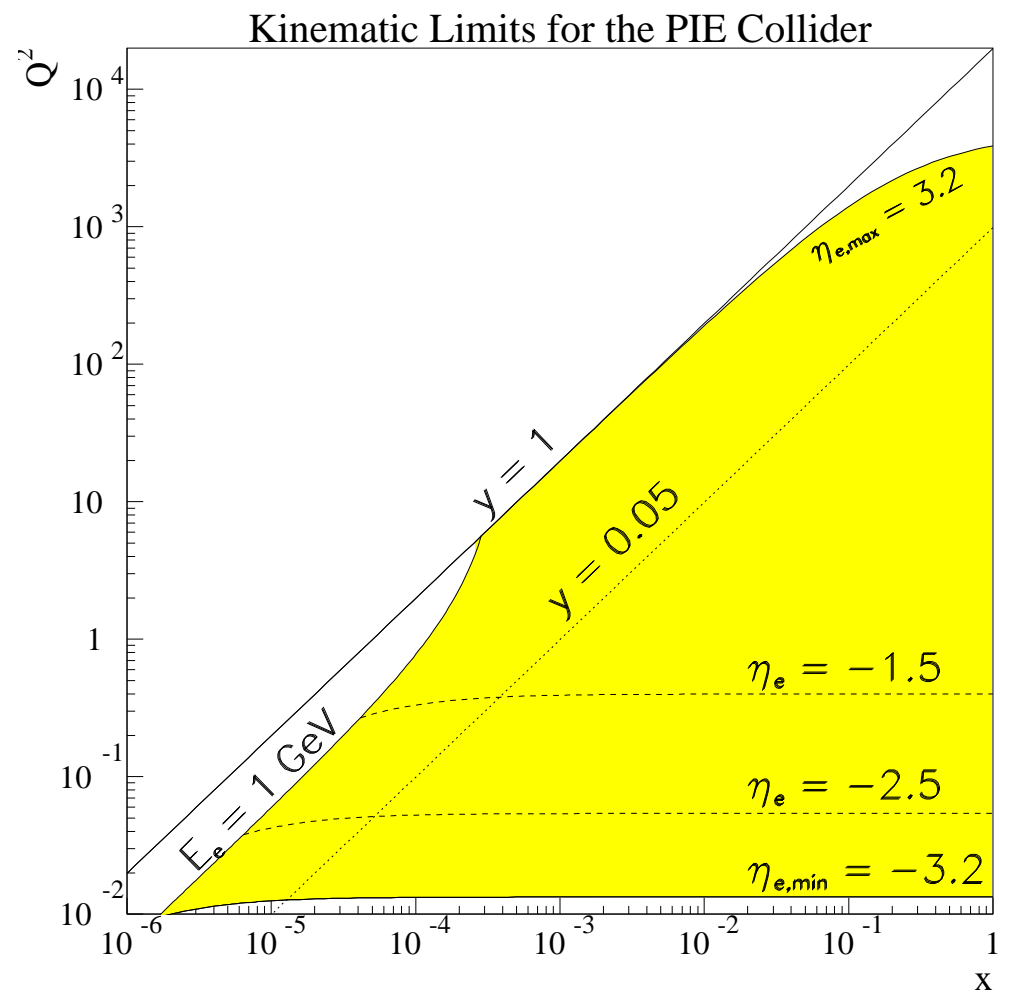

Figure 4: Kinematic domain of the PIE collider - the pseudorapidity acceptance for the electron-proton collisions.

The covered region extends over 5 orders of magnitude in $x_{B j}$ and $Q^{2}$ and over 3 orders of magnitude in the perturbative-QCD region. The measurement domain is 
limited by the pseudorapidity $\eta= \pm 3.2$ lines reflecting the geometrical acceptance limit of measuring the scattered electron in the ATLAS detector [12], and by the $E_{e}=1 \mathrm{GeV}$ line reflecting the minimum energy at which the electrons can be comfortably identified and measured.

In order to illustrate the achievable resolution in $x_{B j}$ the $y=0.05$ line is drawn. Below this line, the $x_{B j}$ resolution is larger than $100 \%$. In the $\left(x, Q^{2}\right)$ domain above the $\eta=-1.5$ line most of scattered electrons will be detected in the high precision barrel detector. It must be stressed, that over the a large part of the kinematic domain the scattered electron carries a very small energy. Even if, from the point of view of the precision of the measurement of the event kinematics, this is can be handled easily by reconstructing the track momentum rather than the electron-cluster energy, triggering of these events will be a challenge. For example - the minimum bias first level trigger will have to be followed by a dedicated, topological, second level trigger [10].

With respect to earlier experiments, the specialty of the PIE@LHC collider, related to high asymmetry of the energy of the electron and the proton beams, is that it covers the small $x_{B j}$ domain for a broad range of the $Q^{2}$ values. This feature of the PIE@LHC provides an alternative to the proposed HERA III programme, which attempts to cover such a domain by extending the measurement of the scattered electrons to very small angles by means of a new detector [13].

\section{Outlook}

The ideas and the calculations presented in this paper need to be followed by a dedicated accelerator expert's analysis. If no "showstoppers" are found, dedicated measurements will have to be made. Even if the decisive feasibility proof of running partially-stripped ion beams at the LHC have to be postponed until the LHC collider is operational, several key tests can be done already now at BNL.

The first, and perhaps the most important one, is to send the low intensity $A u^{77+}$ beam to the RHIC and to measure its life time. This operation requires refraining from stripping of the last two electrons which takes place in the transfer line between AGS and RHIC.

If stable beams are observed, and if the $A u^{79+}-d$ collision runs at the RHIC are scheduled again, then it would be highly desirable to replace the $A u^{79+}$ by the $A u^{77+}$ ions. This exercise could determine the luminosity lifetime for collisions of partially-stripped ion beam and would allow a comparison of the emittance of the $A u^{77+}$ beam with that of the $A u^{79+}$ beam at various stages of the beam acceleration in the RHIC complex.

A dedicated investigation of the proposed beam cooling method could involve emittance studies of the AGS $A u^{77+}$ beam and studies of its evolution in the RHIC 


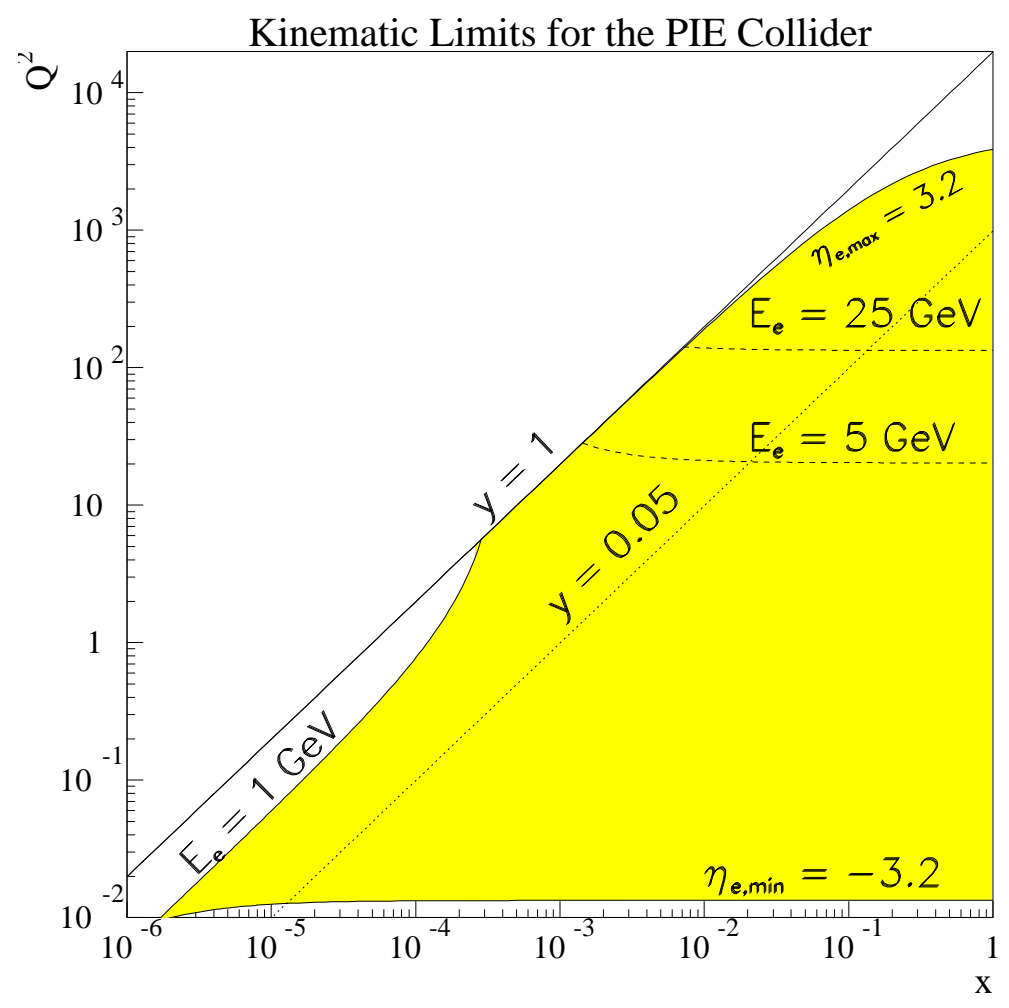

Figure 5: Kinematic domain of the PIE collider - the scattered-electron energies for the electron-proton collisions.

ring, as a function of the acceleration rate. If a reduction of the transverse normalized emittance is observed, then the next step would be to try to understand the beam-cooling mechanism by a direct detection of monochromatic photons emitted in radiative transitions of thermally excited gold ions. The characteristic photon energies of the atomic de-excitations of gold ions are expected to be Doppler-shifted by a $\gamma$ factor reflecting the acceleration phase of the beam. The detector of these photons should cover the energy range of $0.5-5 \mathrm{MeV}$ and the angular range of $0 \leq \theta \leq \gamma$ collimated along the direction of the beam of partially stripped ions.

Observing the $\gamma$ dependence of the rate of the monochromatic photons could identify the characteristic acceleration phase in which the bunch-temperature stabilization takes place. Last, but not the least, an experimental investigation of the light emission in the process of the iso-thermal heat evaporation could shed some light on the mechanics the light emission in supernova explosions. 
Even if an effective spontaneous cooling of the $A u^{77+}$ bunches is observed such a beam cannot be used in $A u^{77+}-A u^{77+}$ collisions because the luminosity lifetime would be too short. Moreover, recording ed collisions in $A u^{77+}-d$ runs would not add much to the scope of the BNL physics programme - the equivalent electron beam energy is simply too low. Why then bother with such studies at BNL? The main reason is that if the beam colling method would work, the gain for the future eRHIC@BNL programme would be of high value - in particular if the option of the dedicated QCD-facility [11] could be realized at BNL. In such a facility low emittance nuclear beams are indispensable for using nuclei as low-noise, femto-detectors capable of studying the space-time evolution of variable partonic configurations at the Fermi distance-scale.

Only if the results of the above tests at BNL would validate the ideas presented in this paper, it would make sense to consider including partially-stripped-ion-runs in the CERN LHC programme, and changing the stripping sequence of ions.

\section{Conclusions}

The preliminary results presented in this paper suggest that electrons attached to the high-Z ions can be transported over the LHC acceleration chain and stored at the top LHC energy.

The hybrid beam of monochromatic electrons and their carrier ions is fragile and can be only used in collisions with protons and fully stripped light ions. The luminosity of electron-proton and electron-light ion collisions, appears to be sufficient to provide a precise, detector-dependent diagnostic of partonic WBBs used in the standard LHC physics programme.

The beam colling method discussed in this paper, if confirmed experimentally, may have an important impact on the acceleration and storage of high energy ion beams and on the future eRHIC project at BNL.

\section{Acknowledgment}

I would like to thank Kai Hencken for drawing my attention to ionization processes as the main limitations for storing partially-stripped ions, to Leif Ahrens, Steve Peggs and Dejan Trbojevic for several valuable suggestions, to Stefan Valkar for discussion of negatively charged hydrogen atoms, to Daniel Brandt, Karl-Heinz Schindl, and John Jowett for their interest in this work, to Janusz Chwastowski and Steve Armstrong for help in preparing the manuscript. 


\section{References}

[1] H. Rausch and V. Traubenberg, Naturwiss. 18, 417 (1930).

[2] C. Lanczos, Z. Physik 62, 518 (1930) and 68, 204 (1931).

[3] J.R. Oppenheimer, Phys. Rev. 31, 66 (1928).

[4] E.G. Bessonov, E.V. Tkalya, arXiv:physics/0212100v1, 27 Dec. 2002.

[5] R. Anholt and U. Becker, Phys. Rev. A36, 4628, (1987).

[6] H.F. Krause et al., Phys. Rev. A63, 63, (2001).

[7] J.M. Jowett, Ions in the LHC Ring, Proceedings of the LHC project Workshop - Chamonix XIII, CERN-AB-2004-014 ADM.

[8] A. Rossi, N. Hilleret, Residual Gas Density Estimation in the LHC Experimental Interaction Regions, LHC Project Report 674 (2003).

[9] I. Abt et al., Nucl.Phys. B407, 515 (1993).

[10] M.W. Krasny, A Model of Dynamic Integration of ATLAS Detector, Trigger and Software in LHC Data-Taking Environment. Note II : A Gauge Model of Data Taking, ATL-COM-GEN-2003-004, CERN, 16 Dec 2003, and references quoted therein.

[11] M.W. Krasny, Nucl.Phys.Proc.Suppl. 105 185, (2002).

[12] ATLAS Detector and Physics Performance, Technical Design Report. CERN/LHCC/99-15, 25 May 1999.

[13] A New Experiment for the HERA Collider. Expression of interest. DESY, April 2003. 